\title{
Article \\ Subfractional Spectrum of Serum Lipoproteins and Gut Microbiota Composition in Healthy Individuals
}

\author{
Daria A. Kashtanova ${ }^{1, *} \mathbb{0}$, Natalia S. Klimenko ${ }^{2} \mathbb{D}$, Olga N. Tkacheva $^{1}$, Irina D. Strazhesko ${ }^{1}$, \\ Victoria A. Metelskaya ${ }^{3}$, Natalia V. Gomyranova ${ }^{4}$ and Sergey A. Boytsov ${ }^{4}$ \\ 1 The "Russian Clinical Research Center for Gerontology" of the Ministry of Healthcare of the Russian \\ Federation, Pirogov Russian National Research Medical University, 16 1st Leonova Str., \\ 129226 Moscow, Russia; tkacheva@rgnkc.ru (O.N.T.); istrazhesko@gmail.com (I.D.S.) \\ 2 Center for Precision Genome Editing and Genetic Technologies for Biomedicine, Institute of Gene Biology, \\ Russian Academy of Sciences, Vavilova Str., 34/5, 119334 Moscow, Russia; natasha.klmnk@gmail.com \\ 3 National Research Center for Therapy and Preventive Medicine of the Ministry of Health of the Russian \\ Federation, 10 Petroverigsky Str., 101990 Moscow, Russia; vametelskaya@gmail.com \\ 4 National Medical Research Center of Cardiology of the Ministry of Health of the Russian Federation, 15a Str. \\ 3rd Cherepkovskaya, 121552 Moscow, Russia; NGomyranova@gnicpm.ru (N.V.G.); \\ prof.boytsov@gmail.com (S.A.B.) \\ * Correspondence: dr.kashtanova@gmail.com
}

\section{check for}

updates

Citation: Kashtanova, D.A.; Klimenko, N.S.; Tkacheva, O.N.; Strazhesko, I.D.; Metelskaya, V.A.; Gomyranova, N.V.; Boytsov, S.A. Subfractional Spectrum of Serum Lipoproteins and Gut Microbiota Composition in Healthy Individuals. Microorganisms 2021, 9, 1461.

https://doi.org/10.3390/

microorganisms 9071461

Academic Editor: Fabio Pace

Received: 26 May 2021

Accepted: 3 July 2021

Published: 8 July 2021

Publisher's Note: MDPI stays neutral with regard to jurisdictional claims in published maps and institutional affiliations.

Copyright: (c) 2021 by the authors. Licensee MDPI, Basel, Switzerland. This article is an open access article distributed under the terms and conditions of the Creative Commons Attribution (CC BY) license (https:// creativecommons.org/licenses/by/ $4.0 /)$
Abstract: Aim: To reveal the relationship between gut microbiota composition and subfractional spectrum of serum lipoproteins and metabolic markers in healthy individuals from Moscow. Methods: The study included 304 participants (104 were men), who underwent thorough preclinical assessment to exclude any chronic disease as well as cardiovascular pathology. Lipoprotein subfractional distribution was analyzed by Lipoprint LDL System (Quantimetrix, Redodno Beach, CA, USA). Gut microbiota composition was assessed by $16 \mathrm{~S}$ rRNA sequencing of V3-V4 regions. Results: High gut microbiota diversity was positively associated with HDL-cholesterol (C) level and negatively associated with abdominal obesity, BMI, and dyslipidemia. According to selbal analysis, excessive representation of Prevotella spp. was positively associated with IDL-C and LDL-2-C. VLDL-C correlated with Ruminococcus_u/Faecalibacterium_prausnitzii balance. An unexpected positive relationship between LDL-C level and Bifidobacteriaceae_u/Christensenellaceae_u to Bifidobacterium_u balance was found, which may reflect the importance of the integrative microbiota assessment. Low microbiota diversity was associated with obesity, abdominal obesity and low HDL-C level. Conclusions: Gut microbiota imbalance may be one of the components involved in metabolic disorders. The balance of microorganisms and the microbiota diversity may play a more significant role in human health than individual bacterial genera.

Keywords: gut microbiota; 16S rRNA sequencing; atherosclerosis; lipoproteins; subfractional analysis of lipoproteins

\section{Introduction}

Cardiovascular events resulting from the formation of atherosclerotic plaques and atherothrombosis continue to be a heavy burden on the healthcare system and the economy worldwide. Despite all efforts made by scientists and physicians, we still do not have any unambiguous understanding of the mechanisms of the development of cardiovascular diseases. Numerous studies have shown that elevated blood levels of low-density lipoproteins (LDL) cholesterol (C), as well as low levels of high-density lipoproteins (HDL)$\mathrm{C}$, are associated with an increased risk of developing symptomatic atherosclerosis [1]. Nevertheless, a significant proportion of patients suffering from cardiovascular disease (CVD) have normal or even reduced LDL-C levels and normal HDL-C concentrations. Traditional CVD risk factors, including dyslipidemia, do not always allow to correctly stratify patients according to the level of individual risk of cardiovascular events. Indeed, 
we can often observe different levels of CVD and different pathways for the development of cardiovascular events in individuals with seemingly similar risk factors according to lipid profile and SCORE scale indicators. Thus, the traditional schemes for determining the risk of CVD developing need further improvement, since the vast majority of coronary events still occur in low and moderate risk groups.

Plasma lipoproteins represent a heterogeneous population of particles varying in density, lipid-protein composition, and size. According to size, LDL are composed of large (LDL1), middle (LDL2), and small dense particles (sdLDL3-7). LDL size seems to be an important predictor of cardiovascular events and progression of CVD. There is increasing evidence that sdLDL are strongly associated with higher CVD risk. A number of studies in recent years have shown that analysis of lipoproteins' subfractional distribution can provide the additional information concerning their function and role in CVD, both as in the case of HDL [2] and LDL [3]. It is worth noting that the main focus of this article is the LDL fractions.

The gut microbiota could play an active role in lipoprotein metabolism. Epidemiological and preclinical studies showed an association between bacterial communities and cholesterolemia. However, this association remains poorly understood and characterized. Studies investigating the relationship between the composition of the gut microbial community and the concentration of cholesterol transported by lipoproteins have shown that the overrepresentation of certain gut bacteria has a close relationship with blood cholesterol levels, and microbiota transplantation from people with dyslipidemia to mice leads in cholesterol levels increase in these animals [4,5]. Other studies report that use of certain bacterial species, such as probiotics, may decrease host cholesterol levels [6]. The ability of microorganisms to convert cholesterol into coprostanol has long been known. Coprostanol is absorbed much less in the intestine. In recent years, studies have indicated that many previously unknown gut microorganisms are involved in this process [7]. Another interesting aptitude of gut microorganisms is their ability to bind cholesterol directly. For example, some intestinal lactobacilli have this property [8]. The same lactobacilli, enterococci, and some bifidobacteria can deconjugate bile acids-the process, which complicates cholesterol absorption [8,9].

Recently we studied gut microbiota of relatively healthy individuals free of CVD manifestations but who had CVD risk factors. We did not find any relationship between lipoprotein levels and microbiota composition according to standard analyzes of lipoproteins using the homogeneous enzymatic colorimetric method [8-10]. For some studied participants, subfractional distribution of apolipoprotein (apo) B-containing lipoproteins was analyzed using by Lipoprint LDL System. The system uses polyacrylamide gel electrophoresis to separate different lipoprotein subfractions by size.

The purpose of the current study was to evaluate the relationship between gut microbiota composition and subfractional profile of serum low densities lipoproteins and their metabolic status in healthy Moscow inhabitants.

\section{Materials and Methods}

\subsection{Recruitment of Study Participants}

The study included Caucasian participants (from Moscow and Moscow Region) aged over 18 years, after the preventive outpatient screening. None of participants had been treated with any drugs (for at least 6 months); nobody had any clinical manifestations of CVD or other chronic diseases. Sex was defined as biological sex. Informed consent was obtained from all subjects involved in the study. Data privacy was ensured by using anonymized identifiers. The study was approved by the local ethics committee, meeting \#8, 29 November 2011. Patients' pre-assessment and exclusion criteria described in detail in the previous paper [10]. Potential participants with any significant deviations in examinations were not included in the study. 


\subsection{Sample Collection and Gut Microbiota Analysis}

Stool samples were frozen and stored at $-20{ }^{\circ} \mathrm{C}$ and thawed before analysis. To assess microbiota composition, sequencing of the $16 \mathrm{~S}$ rRNA gene variable V3-V4 regions was performed (subsequent to total DNA isolation and library preparation) by using the MiSeq Reagent Kit v2 and MiSeq sequencer (Illumina, San Diego, California, CA, USA) according to Illumina recommendations. Libraries were prepared with '16S Metagenomic Sequencing Library Preparation: Preparing 16S Ribosomal RNA Gene Amplicons for the Illumina MiSeq System' protocol (15044223 Rev. B) using the Nextera XT Index Kit (Illumina) with a dual indexing strategy.

\subsection{Lipoproteins Subfractional Spectrum Analysis}

Lipoprotein subfractional distribution was analyzed by Lipoprint LDL System (Quantimetrix, Manhattan Beach Boulevard Redondo Beach, CA, USA). Separation of lipoprotein particles by size was carried out by 3\% polyacrylamide gel electrophoresis (PAGE) in tubes with subsequent scanning and analysis using Lipoware Analysis Program. This method allows to separate lipoproteins subfractions without their preliminary processing with determination the portion of each subfraction (in percent) and the amount of cholesterol (in $\mathrm{mg} / \mathrm{dl}$ ) in the following lipoproteins: very-low-density lipoprotein (VLDL), intermediate-density lipoprotein (IDL) IDL-A, IDL-B, IDL-C, LDL-1, LDL-2, LDL-3, LDL-4, LDL-5, LDL-6, LDL-7, and HDL.

\subsection{Intima-Media Thickness Assessment}

Intima-media thickness (IMT) assessment was carried out using Q-LAB (Philips, The Netherlands) carotid artery duplex scan in B-mode with ECG recording. IMT $<0.9 \mathrm{~mm}$ was considered to be normal; $0.9-1.3 \mathrm{~mm}$ indicated increased thickness. Atherosclerosis was defined as IMT $>1.3 \mathrm{~mm}$ or a local increase in IMT of $0.5 \mathrm{~mm}$ or a $50 \%$ increase in nearby IMT. Plaque was considered as a local IMT thickening higher than $1.0 \mathrm{~mm}$, which caused lumen stenosis but did not affect its internal anatomy.

\subsection{Bioinformatics Analysis}

The primary processing of $16 \mathrm{~S}$ rRNA gene sequencing data was carried out using the Knomics-Biota platform [11] using closed-reference OTU picking with Qiime 1.9 [12] and the GreenGenes [13] reference base with preliminary cropping and filtering of lowquality reads. Alpha diversity was calculated using the chao1 and Shannon metrics. Alpha diversity measures summarize the structure of the ecological community in terms of its number of taxonomic groups as well as its distribution.

\subsection{Statistical Analysis}

The differences of clinical characteristics between groups of patients were assessed using Student's $t$ test. The dependency between intima-media thickness and lipoprotein subfractions was calculated using a linear model. Multiple comparison correction in both analyses was conducted with Benjamini-Hochberg method.

The search for associations between metadata and microbiota composition was carried out on the level of species using the selbal package [13,14]. This package is suitable for working with compositional data, it implements an algorithm that searches for the ratio of bacterial abundances that may predict the factor of interest. The following linear model was analyzed with selbal algorithm: balance age + sex + factor (in case of abdominal obesity, which is a binary factor-logistic regression). The selbal algorithm includes crossvalidation. We considered reliable the results where the bacteria were selected to the best balance in more than $50 \%$ of cross-validation iterations and at the same time the final model $\mathrm{R} 2>0.2$.

Additionally, the associations between each bacterium and metadata were analyzed using linear model: taxon_abundance $\sim$ age + sex + factor. For this analysis taxon abundance was preliminary clr-transformed, zero values were imputed with Bayesian-multiplicative re- 
placement (cmultRepl function from the package zCompositions [15]. The multiple comparison correction was performed on each taxonomic level using Benjamini-Hochberg method.

Associations between alpha-diversity of the microbial community and metadata were assessed using linear model: diversity $\sim$ age + sex + factor. The multiple comparison correction was performed using Benjamini-Hochberg method.

\section{Results}

The study included 304 participants aged $52 \pm 13$ y.o. The clinical characteristics are shown in Table 1. As one can see from this Table, age risk group (men aged $\geq 45$ y.o. and women aged $\geq 55$ ) had more risk factors, such as higher body mass index (BMI), systolic blood pressure (SBP), Atherogenic index of plasma (AIP) and glucose level, as well as bigger waist circumference. Men were younger than women. Despite this, with false discovery rate (FDR) adjustment, men were more likely to have some risk factors, which is quite common. In general, we can say that the distribution of risk factors is also quite typical.

We analyzed a total of 12 lipoprotein subfractions, including VLDL, three IDL (IDL-A, IDL-B, and IDL-C) subfractions, seven LDL subfractions and HDL. Levels of lipoproteins $(\mathrm{mg} / \mathrm{dL})$ subfractions are presented in Table 2.

Besides HDL-C, which is commonly higher in women, VLDL-C differed significantly between men and women and was much higher in men. It is known that men sex is a risk factor for the onset of cardiovascular events, which is also aggravated by a less favorable profile of cholesterol subfractions. Levels of cholesterol in IDL-B, IDL-A, LDL-2, and total LDL-C were higher in the aged group. All these subfractions can be considered as potentially pro-atherogenic.

As a next step, we analyzed lipoprotein subfractional distribution in subjects who were underwent carotid artery dopplerography by means of linear regression. According to the results, higher VLDL and IDL-B cholesterol levels were associated with intima-media thickening (Table 3). It is noteworthy that none of the other studied subfractions showed their significance not only after the correction for multiple comparisons, but also in the primary analysis.

\subsection{Gut Microbiota Composition}

All samples passed the quality control of classified reads proportion (all samples contained at least $70 \%$ of classified reads). Gut microbiota composition was quite similar to that in other studies [16-18]. The most presented bacteria were Clostridiales, Ruminococcaceae, Bacteroides and some others (Figure 1). Such composition is normally observed among residents of Western countries, and correlates with the data of literary sources. Detailed interactive visualizations of microbiome characteristics are available here https:/ / biota.knomics.ru/lipids-healthy-individuals (accessed on 19 June 2021). 
Table 1. Clinical characteristics of the studied participants.

\begin{tabular}{|c|c|c|c|c|c|c|c|c|c|}
\hline & $\begin{array}{l}\text { Total Cohort } \\
\quad(n=304)\end{array}$ & $\begin{array}{c}\text { Males } \\
(n=104)\end{array}$ & $\begin{array}{l}\text { Females } \\
(n=200)\end{array}$ & $p$-Value & FDR & $\begin{array}{c}\text { Young } \\
(n=139)\end{array}$ & $\begin{array}{c}\text { Age Risk Group } \\
\quad(n=165)\end{array}$ & $p$-Value & FDR \\
\hline Age, years & $51.5 \pm 13.26$ & $48.1 \pm 12.03$ & $53.27 \pm 13.55$ & 0.0008 & 0.0014 & $40.45 \pm 8.55$ & $60.82 \pm 8.52$ & - & - \\
\hline BMI, $\mathrm{kg} / \mathrm{m}^{2}$ & $27.36 \pm 5.14$ & $28.59 \pm 4.22$ & $26.72 \pm 5.46$ & 0.0011 & 0.0017 & $26.5 \pm 5.55$ & $28.08 \pm 4.67$ & 0.0086 & 0.0118 \\
\hline $\mathrm{SBP}, \mathrm{mmHg}$ & $125.33 \pm 16.42$ & $129.52 \pm 14.61$ & $123.14 \pm 16.91$ & 0.0007 & 0.0014 & $120.09 \pm 15.26$ & $129.74 \pm 16.11$ & $<0.00001$ & $<0.00001$ \\
\hline $\mathrm{DBP}, \mathrm{mmHg}$ & $78.16 \pm 10.26$ & $79.88 \pm 10.16$ & $77.26 \pm 10.22$ & 0.0342 & 0.0376 & $77.24 \pm 10.39$ & $78.93 \pm 10.12$ & 0.1555 & 0.1711 \\
\hline Smokers, $\mathrm{n}$ & $58(19 \%)$ & $30(29 \%)$ & $28(14 \%)$ & 0.0171 & 0.0210 & 27 (19\%) & 31 (19\%) & 1.0000 & 1.0000 \\
\hline $\mathrm{TC}, \mathrm{mmol} / \mathrm{L}$ & $5.65 \pm 1.14$ & $5.61 \pm 1.02$ & $5.68 \pm 1.21$ & 0.5958 & 0.5958 & $5.4 \pm 0.97$ & $5.86 \pm 1.24$ & 0.0003 & 0.0005 \\
\hline $\mathrm{TG}, \mathrm{mmol} / \mathrm{L}$ & $1.28 \pm 0.85$ & $1.6 \pm 1.07$ & $1.12 \pm 0.65$ & $<0.00001$ & 0.0001 & $1.09 \pm 0.68$ & $1.45 \pm 0.94$ & 0.0001 & 0.0002 \\
\hline AIP & $-0.07 \pm 0.72$ & $0.31 \pm 0.72$ & $-0.27 \pm 0.64$ & $<0.00001$ & $<0.00001$ & $-0.27 \pm 0.71$ & $0.09 \pm 0.7$ & $<0.00001$ & $<0.00001$ \\
\hline Fasting plasma glucose, $\mathrm{mmol} / \mathrm{L}$ & $5.75 \pm 1.4$ & $6.1 \pm 1.61$ & $5.56 \pm 1.24$ & 0.0036 & 0.0049 & $5.3 \pm 0.94$ & $6.12 \pm 1.6$ & $<0.00001$ & $<0.00001$ \\
\hline WC, $\mathrm{cm}$ & $89.54 \pm 15.3$ & $98.67 \pm 12.46$ & $84.84 \pm 14.5$ & $<0.00001$ & $<0.00001$ & $85.17 \pm 15.01$ & $93.21 \pm 14.59$ & $<0.00001$ & $<0.00001$ \\
\hline
\end{tabular}

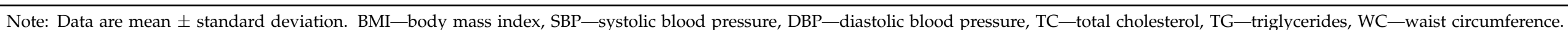

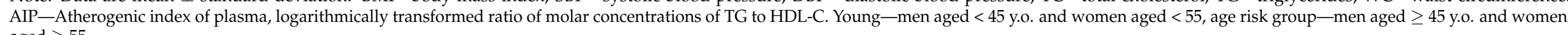
aged $\geq 55$.

Table 2. Lipoproteins subfractions (mg/dl), sex and age.

\begin{tabular}{|c|c|c|c|c|c|c|c|c|c|}
\hline Cholesterol, mg/dL & Total Cohort $(n=304)$ & $\begin{array}{c}\text { Males } \\
(n=104)\end{array}$ & $\begin{array}{l}\text { Females } \\
(n=200)\end{array}$ & $p$-Value & FDR & $\begin{array}{c}\text { Young } \\
(n=139)\end{array}$ & $\begin{array}{l}\text { Age Risk Group } \\
\quad(n=165)\end{array}$ & $p$-Value & FDR \\
\hline VLDL & $15.49 \pm 4.44$ & $17.14 \pm 4.10$ & $14.68 \pm 4.39$ & 0.0002 & 0.0012 & $14.87 \pm 4.29$ & $16.36 \pm 4.53$ & 0.0223 & 0.0580 \\
\hline IDL-C & $23.08 \pm 8.32$ & $23.49 \pm 7.66$ & $22.88 \pm 8.65$ & 0.6225 & 0.6744 & $22.38 \pm 8.17$ & $24.08 \pm 8.48$ & 0.1662 & 0.2700 \\
\hline IDL-B & $18.92 \pm 5.88$ & $18.89 \pm 4.34$ & $18.93 \pm 6.53$ & 0.9644 & 0.9644 & $17.39 \pm 4.86$ & $21.08 \pm 6.52$ & $<0.00001$ & 0.0002 \\
\hline IDL-A & $21.34 \pm 8.09$ & $20.38 \pm 8.47$ & $21.82 \pm 7.89$ & 0.2569 & 0.4564 & $20.04 \pm 7.77$ & $23.18 \pm 8.22$ & 0.0084 & 0.0273 \\
\hline LDL-1 & $39.77 \pm 13.7$ & $38.08 \pm 12.68$ & $40.61 \pm 14.15$ & 0.2109 & 0.4564 & $38 \pm 11.84$ & $42.27 \pm 15.71$ & 0.0417 & 0.0902 \\
\hline LDL-2 & $18.8 \pm 12.27$ & $21.17 \pm 11.99$ & $17.63 \pm 12.28$ & 0.0573 & 0.2484 & $16.2 \pm 11.23$ & $22.48 \pm 12.79$ & 0.0006 & 0.0024 \\
\hline LDL-3 & $3.65 \pm 5.00$ & $4.61 \pm 5.82$ & $3.17 \pm 4.49$ & 0.0853 & 0.2773 & $3.19 \pm 4.88$ & $4.3 \pm 5.12$ & 0.1310 & 0.2434 \\
\hline LDL-5 & $0.08 \pm 0.88$ & $0.20 \pm 1.50$ & $0.02 \pm 0.18$ & 0.3234 & 0.4564 & $0.12 \pm 1.14$ & $0.01 \pm 0.11$ & 0.3055 & 0.3776 \\
\hline LDL-6 & $0.01 \pm 0.14$ & $0.03 \pm 0.25$ & $0.00 \pm 0.00$ & 0.3211 & 0.4564 & $0.02 \pm 0.19$ & $0 \pm 0$ & 0.3195 & 0.3776 \\
\hline LDL-7 & $0.21 \pm 2.40$ & $0.03 \pm 0.25$ & $0.30 \pm 2.93$ & 0.2984 & 0.4564 & $0.02 \pm 0.19$ & $0.49 \pm 3.71$ & 0.2618 & 0.3776 \\
\hline Total LDL & $127.23 \pm 34.74$ & $130.20 \pm 27.56$ & $125.75 \pm 37.84$ & 0.3551 & 0.4564 & $118.26 \pm 29.49$ & $140.08 \pm 37.72$ & $<0.00001$ & 0.0002 \\
\hline HDL & $56.16 \pm 15.3$ & $47.83 \pm 13.21$ & $60.29 \pm 14.61$ & $<0.00001$ & $<0.00001$ & $56.94 \pm 14.19$ & $55.05 \pm 16.78$ & 0.4135 & 0.4480 \\
\hline
\end{tabular}

Note: Young-men aged $<45$ y.o. and women aged $<55$, Age risk group—-men aged $>45$ y.o. and women aged $>55$. 
Table 3. Cholesterol content in lipoprotein subfractions and intima-media thickness $(\mathrm{N}=287)$.

\begin{tabular}{cccc}
\hline Cholesterol, mg/dL & $\begin{array}{c}\text { Linear Regression } \\
\text { Coefficient }\end{array}$ & $\boldsymbol{p}$-Value & $\begin{array}{c}\text { FDR Adjusted } \\
\boldsymbol{p} \text {-Value }\end{array}$ \\
\hline VLDL & 0.0136 & $<0.00001$ & 0.0001 \\
IDL-C & 0.0035 & 0.0324 & 0.0789 \\
IDL-B & 0.0080 & 0.0004 & 0.0025 \\
IDL-A & 0.0016 & 0.3564 & 0.4633 \\
LDL-1 & -0.0011 & 0.2864 & 0.4137 \\
LDL-2 & 0.0025 & 0.0237 & 0.0769 \\
LDL-3 & 0.0056 & 0.0364 & 0.0789 \\
LDL-4 & 0.0089 & 0.1543 & 0.2508 \\
LDL-5 & -0.0022 & 0.8844 & 0.8844 \\
LDL-6 & -0.0282 & 0.7638 & 0.8275 \\
LDL-7 & 0.0098 & 0.0801 & 0.1487 \\
Total LDL & 0.0009 & 0.0173 & 0.0751 \\
HDL & -0.0004 & 0.6894 & 0.8147 \\
\hline
\end{tabular}

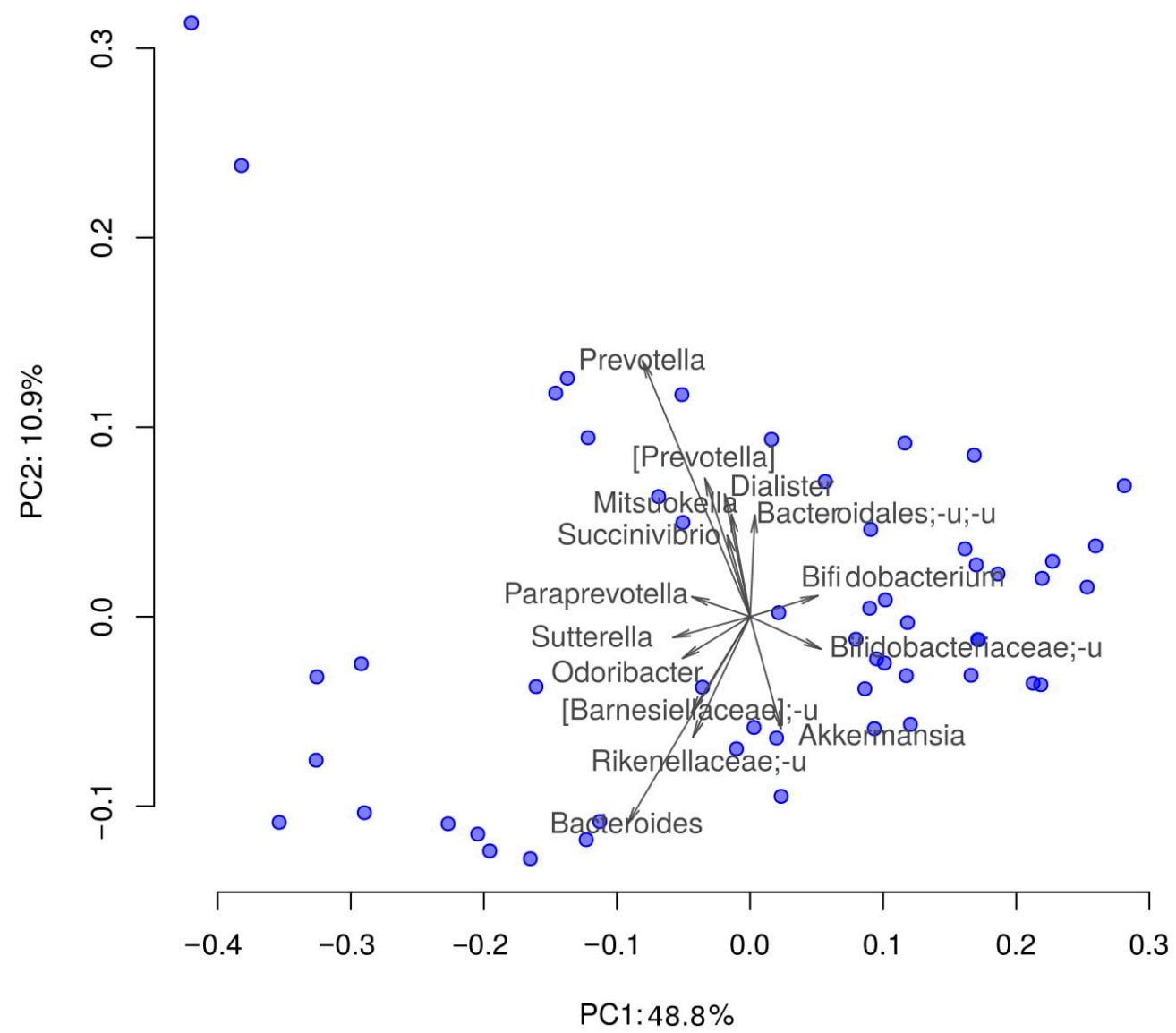

Figure 1. Distribution of the samples by their taxonomic composition in reduced dimensionality. PCoA (Principal Coordinate Analysis); dissimilarity metric: weighted UniFrac. Prefix "_u" denotes all unclassified taxa from the higher taxonomic rank. Arrows show the top 15 taxa in terms of the explained variance in given axes. The arrows' length is proportional to the percent of variance explained by the taxon. The arrows' angle reflects the distribution of this variance between the axes.

\subsection{Association between the Gut Microbiota Composition and Subfractional Spectrum of Apo} $B$-Containing Lipoproteins

First, it should be pointed that there were no associations between gut microbiota composition and lipid spectrum when linear model approach after clr transformation was applied. Another analysis variant was selbal algorithm and it revealed a number of 
reliable associations (Figure 2$)$ : reproducible ( $>50 \%$ of cross-validation iterations included a taxon) and with good prediction quality (R2 of a final model was higher than 0.2) (Table 4). Selbal algorithm operates with microbial balances. Microbial balances are specific microbiome features that allow to perform differential abundance analysis in a compositionality-aware way. Balance is proportional to the log ratio between geometric means of two bacteria groups denoted numerator and denominator [14]. The choice of methods using balances is justified by the fact that the gut microbiota is a complexly organized community. The analysis of individual microbe as a rule is effective only when one microorganism is the causative agent of the disease. On the contrary, balances allow to perform a more comprehensive approach to assessing the relationship between the microbiota and the host health state.

Among clinical parameters, BMI was associated positively with unclassified species from [Prevotella] genus and Enterobacteriaceae family, and negatively-with unclassified genera from Clostridiaceae family. Unclassified species from [Prevotella] genus were also included in reliable balances for IDL-C and LDL-2 as a numerator (positive association). Interestingly, at the same time IDL-C was negatively related to Prevotella copri abundance.

Unclassified species from Christentsenella genus and Bifidobacteriaceae family were positively associated with LDL-C concentrations while other unclassified species from Bifidobacterium genus-negatively. VLDL-C was negatively related to Faecalibacterium prausnitzii abundance and positively_to unclassified species from Ruminococcus genus.

Table 4. Relationship between the gut microbiota composition, subfractional spectrum of serum apo-B containing lipoproteins and BMI, selbal test results.

\begin{tabular}{|c|c|c|c|c|c|}
\hline Factor & Full Balance & Adjusted $R^{2}$ & Reproducible Taxon & $\begin{array}{l}\text { Direction of } \\
\text { Association }\end{array}$ & $\begin{array}{c}\text { Percent of Times } \\
\text { Included in a } \\
\text { Balance }\end{array}$ \\
\hline \multirow{3}{*}{ BMI } & [Prevotella]_u | & \multirow{3}{*}{0.26515} & [Prevotella]_u & + & 72.5 \\
\hline & Enterobacteri- & & Enterobacteriaceae_u & + & 70 \\
\hline & aceae_u)/Clostridiaceae_u & & Clostridiaceae_u & - & 80 \\
\hline \multirow{3}{*}{ LDL } & Bifidobacteriaceae_u & \multirow{3}{*}{0.26381} & Bifidobacteriaceae_u & + & 87.5 \\
\hline & | Christensenellaceae_u / & & Christensenellaceae_u & + & 92.5 \\
\hline & Bifidobacterium_u & & Bifidobacterium_u & - & 90 \\
\hline \multirow{2}{*}{ VLDL } & Ruminococcus_u/ & \multirow[b]{2}{*}{0.29299} & Ruminococcus_u & + & 50 \\
\hline & Faecalibacterium_prausnitzii & & Faecalibacterium_prausnitzii & - & 75 \\
\hline \multirow{2}{*}{ IDL-C } & \multirow{2}{*}{ [Prevotella]_u/Prevotella_copri } & \multirow{2}{*}{0.32018} & [Prevotella]_u & + & 92.5 \\
\hline & & & Prevotella_copri & - & 75 \\
\hline LDL-2 & $\begin{array}{c}\text { [Prevotella]_u/ } \\
\text { [Ruminococcus]_gnavus }\end{array}$ & 0.28927 & [Prevotella]_u & + & 60 \\
\hline
\end{tabular}

Note: Postfix "_u" denotes all unclassified taxa from the higher taxonomic rank. Note: We considered significant only those taxa from the full balances for which a high reproducibility was observed $(>50 \%)$.

\subsection{Association between Gut Microbiota Diversity and Metabolic Factors}

Remarkable results have been obtained in assessing the diversity of the gut microbiota. Gut microbiota diversity was positively associated with HDL-C level and negatively associated with abdominal obesity, BMI, and AIP (Table 5). Being an extremely important indicator of microbiota well-being, diversity had also been found to be associated with the favorable lipoprotein profile and metabolic health in general.

It should be noted that both low chao and Shannon indexes were positively correlated with obesity as well as abdominal obesity. In contrast, only Shannon index had negative association with AIP and positive-with HDL-C level.

Thus, the bioinformatics analysis revealed the relationship between the general state of the gut microbiota and human health as well as associations of the balance of microorganisms with individual indicators. 


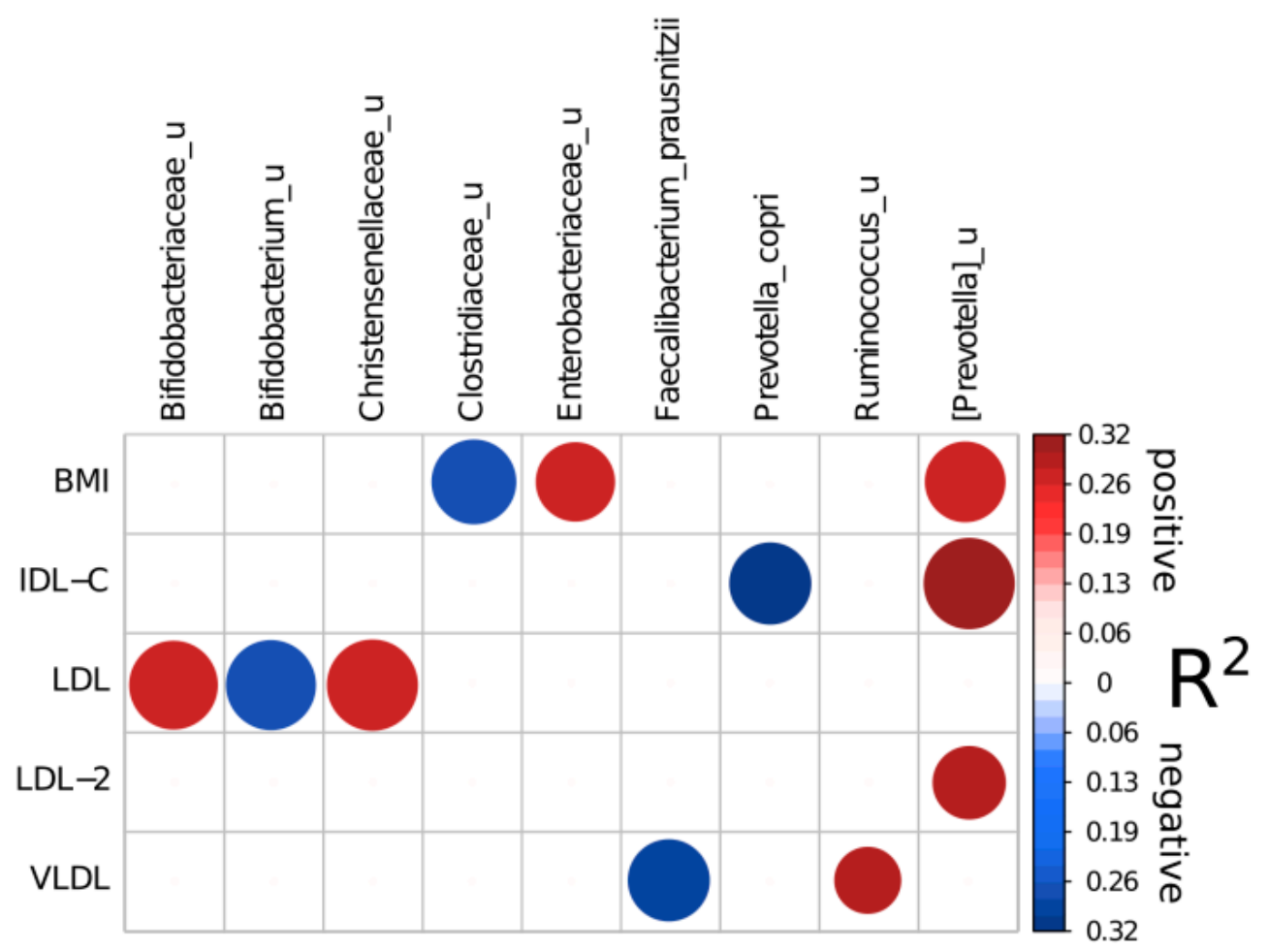

Figure 2. Reliable associations between microbiome composition, subfractional spectrum of apo B-containing lipoproteins and BMI according to Selbal analysis (see Section 2). Circles sizes are related to taxon reproducibility and circles colors to model R2. Numerator parts of balance are colored with red and denominator with blue.

Table 5. Gut microbiota diversity association with clinical parameters.

\begin{tabular}{lllll}
\hline & $p$-Value & $\begin{array}{c}\text { Linear Model } \\
\text { Coefficient }\end{array}$ & FDR & $\begin{array}{c}\text { Alpha Diversity } \\
\text { Metric }\end{array}$ \\
\hline BMI & 0.0017 & -17.5031 & 0.0250 & Chao1 \\
$\begin{array}{l}\text { Abdominal } \\
\text { obesity }\end{array}$ & 0.0083 & -194.5565 & 0.0622 & Chao1 \\
BMI & 0.0029 & -0.0344 & 0.0147 & Shannon \\
$\begin{array}{l}\text { Abdominal } \\
\text { obesity }\end{array}$ & 0.0018 & -0.3968 & 0.0138 & Shannon \\
AIP & 0.0015 & -0.3065 & 0.0138 & Shannon \\
HDL-C & 0.0050 & 0.6334 & 0.0187 & Shannon \\
\hline
\end{tabular}

\section{Discussion}

Metabolic disorders are the most widespread chronic risk factors in the modern world. To this day, their representation continues to grow, and imbalance in gut microbiota is supposed to be one of the mechanisms for the development of such disorders. In the framework of this study, relationships between gut microbiota with lipid spectrum and some metabolic parameters were found.

Human blood cholesterol level is determined by endogenous cholesterol synthesized mainly in liver and exogenous cholesterol obtained from food components of animal origin. Cholesterol synthesized in hepatocytes is transported to the gallbladder and then secreted into the small intestine along with bile salts. In intestine, biliary cholesterol is mixed with dietary cholesterol, and together they are transported into enterocytes for packaging in chylomicrons and secretion into the blood $[15,19]$. The role of gut microbiota in these processes is being actively studied, but so far just a few studies have been carried out on the relationship between the results of sequencing of the gut microbiota and lipoprotein 
subfractions assessment. Serum lipoproteins are represented by a heterogeneous spectrum of particles differing in origin as well as density, size, composition, and functional activity. The main subfractions of lipoproteins are VLDL, IDL (three subfractions C, B, A), large physiologically active LDL-1 and smaller LDL-2 subclasses, small dense LDL-3 to LDL-7, and HDL. Small dense LDL-3-7 subfractions seem to be the most atherogenic, high portion of these particles in the LDL spectrum is associated with the increased risk of atherosclerotic CVD, even if total LDL-C level is normal [20].

In the studied cohort, men had more risk factors than women and age of participants was associated with higher blood pressure, lipids and glucose levels as well as with obesity, including the abdominal obesity. The amount of cholesterol in some lipid fractions such as IDL-B, IDL-A, total LDL and LDL-2 was significantly higher in the age risk group. According to the recent studies, high IDL-C as well as LDL-C concentrations can be associated with coronary atherosclerosis or carotid intima-media thickness. IDL subfractions metabolized to LDL, which are taken up by the LDL receptor in numerous tissues [21]. Cholesterol level in VLDL and IDL-B was also associated with intima-media thickening in our study.

The microbiome composition was associated with metabolic profile. Despite the small group, some bacteria balances were associated with the lipid spectrum. Thus, such unfavorable microbial genera as Enterobacteriaceae_u and poorly characterized [Prevotella]_u were highly represented in obese participants. It is interesting that the same unclassified [Prevotella]_u genus was positively associated with cholesterol content in IDL-C and LDL-2 fractions. Prevotella is quite a common genus in the gut, although it may be harmful due to its potential ability to stimulate inflammation [22]. Role of Prevotella spp. within the gut microbiota as well as their effect on the host are not completely understood. Studies show that Prevotella could activate Toll-like receptors, leading to production of inflammatory cytokines [23]. Notably, high representation of Prevotella was found to be associated with insulin-resistance [22], obesity [24], hypertension [25], as well as with non-alcoholic fatty liver disease [26] in case-control studies. In contrast, beneficial bacteria were reduced in those who had high levels of atherogenic lipoproteins. Faecalibacterium_prausnitzii is linked to healthy plant-based foods and produces butyrateone of the most effective anti-inflammatory agents [26,27].

On the other hand, by means of Selbal analysis we found some unexpected "balances" associated with the lipid spectrum. The balance of Bifidobacteriaceae_u I Christensenellaceae_u and Bifidobacterium_u was associated with total LDL-C level. All these microorganisms are poorly studied, and therefore it is difficult to assess their metabolic profile. Bifidobacteriaceae is a known probiotic taxon. Nevertheless, such association may indicate that the balance of the bifidobacteria community may be no less important than their overall representation. Finally, we have found that the gut microbiota diversity may reflect a normal metabolic status. Low diversity was associated with obesity, abdominal obesity, and dyslipidemia. Our observation that low alpha diversity may correlate with high BMI is consistent with many studies [26-28]. Thus, low diversity and dysbalance of the gut microbiota observed in the adult gut may be considered an indicator of metabolic disturbances even in apparently healthy individuals. Low gut microbiota diversity is also considered to be associated with many other conditions, such as inflammatory bowel disease, psoriatic arthritis, diabetes, and arterial stiffness [29].

\section{Conclusions}

The present study has some limitations, the most important of which is the small size of the cohort. It remains imperative to elucidate the routes and mechanisms that may underlie the microbes and host interaction. Nevertheless, we hope that our results could facilitate prospective studies investigating diverse aspects of gut microbiota influence on human health. Although the characterization of "microbial dark matter" still presents serious barriers, the combination of bioinformatics and biochemical approaches may provide access to this largely untapped source of biologically significant metabolic transformations. 
Author Contributions: D.A.K.-Writing—original draft, Investigation, Methodology, N.S.K.Visualization, Writing - original draft, Formal Analysis, Data curation, O.N.T.-Conceptualization, Project administration, I.D.S.-Project administration, Writing-review and editing, Supervision, V.A.M.-Writing-review and editing, N.V.G.-Writing-review and editing, and S.A.B.Conceptualization, Project administration. All authors have read and agreed to the published version of the manuscript.

Funding: Study was performed at the expense of the center's own funds.

Institutional Review Board Statement: The study was conducted according to the guidelines of the Declaration of Helsinki, and approved by the Ethics Committee of National Medical Research Center for Therapy and Preventive Medicine, meeting \#8, 29 November 2011.

Informed Consent Statement: Informed consent was obtained from all subjects involved in the study.

Data Availability Statement: The data presented in this study are openly available in https:/ /biota. knomics.ru/lipids-healthy-individuals (accessed on 19 June 2021).

Conflicts of Interest: Authors declared no conflict of interest.

\section{References}

1. Mach, F.; Baigent, C.; Catapano, A.L.; Koskinas, K.C.; Casula, M.; Badimon, L.; Chapman, M.J.; De Backer, G.G.; Delgado, V.; Ference, B.A.; et al. 2019 ESC/EAS Guidelines for the Management of Dyslipidaemias: Lipid Modification to Reduce Cardiovascular Risk. Atherosclerosis 2019, 290. [CrossRef]

2. Li, J.-J.; Zhang, Y.; Li, S.; Cui, C.-J.; Zhu, C.-G.; Guo, Y.-L.; Wu, N.-Q.; Xu, R.-X.; Liu, G.; Dong, Q.; et al. Large HDL Subfraction But Not HDL-C Is Closely Linked with Risk Factors, Coronary Severity and Outcomes in a Cohort of Nontreated Patients With Stable Coronary Artery Disease: A Prospective Observational Study. Medicine 2016, 95. [CrossRef]

3. Hernáez, Á.; Soria-Florido, M.T.; Schröder, H.; Ros, E.; Pintó, X.; Estruch, R.; Salas-Salvadó, J.; Corella, D.; Arós, F.; Serra-Majem, L.; et al. Role of HDL Function and LDL Atherogenicity on Cardiovascular Risk: A Comprehensive Examination. PLoS ONE 2019, 14, e0218533. [CrossRef]

4. Le Roy, T.; Lécuyer, E.; Chassaing, B.; Rhimi, M.; Lhomme, M.; Boudebbouze, S.; Ichou, F.; Haro Barceló, J.; Huby, T.; Guerin, M.; et al. The Intestinal Microbiota Regulates Host Cholesterol Homeostasis. BMC Biol. 2019, 17, 94. [CrossRef] [PubMed]

5. Rothschild, D.; Weissbrod, O.; Barkan, E.; Kurilshikov, A.; Korem, T.; Zeevi, D.; Costea, P.I.; Godneva, A.; Kalka, I.N.; Bar, N.; et al. Environment Dominates over Host Genetics in Shaping Human Gut Microbiota. Nature 2018, 555, 210-215. [CrossRef] [PubMed]

6. Park, S.; Kang, J.; Choi, S.; Park, H.; Hwang, E.; Kang, Y.; Kim, A.; Holzapfel, W.; Ji, Y. Cholesterol-Lowering Effect of Lactobacillus Rhamnosus BFE5264 and Its Influence on the Gut Microbiome and Propionate Level in a Murine Model. PLoS ONE 2018, 13, e0203150. [CrossRef]

7. Antharam, V.C.; McEwen, D.C.; Garrett, T.J.; Dossey, A.T.; Li, E.C.; Kozlov, A.N.; Mesbah, Z.; Wang, G.P. An Integrated Metabolomic and Microbiome Analysis Identified Specific Gut Microbiota Associated with Fecal Cholesterol and Coprostanol in Clostridium Difficile Infection. PLOS ONE 2016, 11, e0148824. [CrossRef] [PubMed]

8. Kriaa, A.; Bourgin, M.; Potiron, A.; Mkaouar, H.; Jablaoui, A.; Gérard, P.; Maguin, E.; Rhimi, M. Microbial Impact on Cholesterol and Bile Acid Metabolism: Current Status and Future Prospects. J. Lipid Res. 2019, 60, 323. [CrossRef]

9. Prete, R.; Long, S.L.; Gallardo, A.L.; Gahan, C.G.; Corsetti, A.; Joyce, S.A. Beneficial Bile Acid Metabolism from Lactobacillus Plantarum of Food Origin. Sci. Rep. 2020, 10, 1165. [CrossRef]

10. Kashtanova, D.A.; Tkacheva, O.N.; Doudinskaya, E.N.; Strazhesko, I.D.; Kotovskaya, Y.V.; Popenko, A.S.; Tyakht, A.V.; Alexeev, D.G. Gut Microbiota in Patients with Different Metabolic Statuses: Moscow Study. Microorganisms 2018, 6, 98. [CrossRef] [PubMed]

11. Efimova, D.; Tyakht, A.; Popenko, A.; Vasilyev, A.; Altukhov, I.; Dovidchenko, N.; Odintsova, V.; Klimenko, N.; Loshkarev, R.; Pashkova, M.; et al. Knomics-Biota-A System for Exploratory Analysis of Human Gut Microbiota Data. BioData Min. $2018,11$. [CrossRef]

12. Caporaso, J.G.; Kuczynski, J.; Stombaugh, J.; Bittinger, K.; Bushman, F.D.; Costello, E.K.; Fierer, N.; Peña, A.G.; Goodrich, J.K.; Gordon, J.I.; et al. QIIME Allows Analysis of High-Throughput Community Sequencing Data. Nat. Methods 2010, 7, 335-336. [CrossRef] [PubMed]

13. DeSantis, T.Z.; Hugenholtz, P.; Larsen, N.; Rojas, M.; Brodie, E.L.; Keller, K.; Huber, T.; Dalevi, D.; Hu, P.; Andersen, G.L. Greengenes, a Chimera-Checked $16 \mathrm{~S}$ rRNA Gene Database and Workbench Compatible with ARB. Appl. Environ. Microbiol. 2006, 72, 5069-5072. [CrossRef] [PubMed]

14. Rivera-Pinto, J.; Egozcue, J.J.; Pawlowsky-Glahn, V.; Paredes, R.; Noguera-Julian, M.; Calle, M.L. Balances: A New Perspective for Microbiome Analysis. MSystems 2018, 3. [CrossRef] [PubMed]

15. Palarea-Albaladejo, J.; Martín-Fernández, J.A. zCompositions-R Package for Multivariate Imputation of Left-Censored Data under a Compositional Approach. Chemom. Intell. Lab. Syst. 2015, 143, 85-96. [CrossRef] 
16. Tyakht, A.V.; Kostryukova, E.S.; Popenko, A.S.; Belenikin, M.S.; Pavlenko, A.V.; Larin, A.K.; Karpova, I.Y.; Selezneva, O.V.; Semashko, T.A.; Ospanova, E.A.; et al. Human Gut Microbiota Community Structures in Urban and Rural Populations in Russia. Nat. Commun. 2013, 4, 1-9. [CrossRef] [PubMed]

17. Rinninella, E.; Raoul, P.; Cintoni, M.; Franceschi, F.; Miggiano, G.A.D.; Gasbarrini, A.; Mele, M.C. What Is the Healthy Gut Microbiota Composition? A Changing Ecosystem across Age, Environment, Diet, and Diseases. Microorganisms 2019, 7, 14. [CrossRef]

18. Giuffrè, M.; Campigotto, M.; Campisciano, G.; Comar, M.; Crocè, L.S. A Story of Liver and Gut Microbes: How Does the Intestinal Flora Affect Liver Disease? A Review of the Literature. Am. J. Physiol. Gastrointest. Liver Physiol. 2020, 318, G889-G906. [CrossRef]

19. Xu, Z.; McClure, S.; Appel, L. Dietary Cholesterol Intake and Sources among U.S Adults: Results from National Health and Nutrition Examination Surveys (NHANES), 2001-2014. Nutrients 2018, 10, 771. [CrossRef]

20. Annema, W.; von Eckardstein, A. High-Density Lipoproteins. Multifunctional but Vulnerable Protections from Atherosclerosis. Circ. J. 2013, 77, 2432-2448. [CrossRef]

21. Feingold, K.R. Introduction to Lipids and Lipoproteins; Feingold, K.R., Anawalt, B., Boyce, A., Chrousos, G., de Herder, W.W., Dhatariya, K., Dungan, K., Grossman, A., Hershman, J.M., Hofland, J., et al., Eds.; MDText.com, Inc.: South Dartmouth, MA, USA, 2021. [PubMed]

22. Larsen, J.M. The Immune Response to Prevotella Bacteria in Chronic Inflammatory Disease. Immunology 2017, 151. [CrossRef]

23. Iljazovic, A.; Roy, U.; Gálvez, E.J.C.; Lesker, T.R.; Zhao, B.; Gronow, A.; Amend, L.; Will, S.E.; Hofmann, J.D.; Pils, M.C.; et al. Perturbation of the Gut Microbiome by Prevotella Spp. Enhances Host Susceptibility to Mucosal Inflammation. Mucosal Immunol. 2020, 14, 113-124. [CrossRef]

24. Hu, H.-J.; Park, S.-G.; Jang, H.B.; Choi, M.-G.; Park, K.-H.; Kang, J.H.; Park, S.I.; Lee, H.-J.; Cho, S.-H. Obesity Alters the Microbial Community Profile in Korean Adolescents. PLoS ONE 2015, 10. [CrossRef]

25. Li, J.; Zhao, F.; Wang, Y.; Chen, J.; Tao, J.; Tian, G.; Wu, S.; Liu, W.; Cui, Q.; Geng, B.; et al. Gut Microbiota Dysbiosis Contributes to the Development of Hypertension. Microbiome 2017, 5. [CrossRef] [PubMed]

26. Forslund, K.; Hildebrand, F.; Nielsen, T.; Falony, G.; Le Chatelier, E.; Sunagawa, S.; Prifti, E.; Vieira-Silva, S.; Gudmundsdottir, V.; Pedersen, H.K.; et al. Disentangling the Effects of Type 2 Diabetes and Metformin on the Human Gut Microbiota. Nature 2015, 528, 262. [CrossRef] [PubMed]

27. Liu, H.; Wang, J.; He, T.; Becker, S.; Zhang, G.; Li, D.; Ma, X. Butyrate: A Double-Edged Sword for Health? Adv. Nutr. 2018,9 , 21. [CrossRef] [PubMed]

28. Sun, L.; Ma, L.; Ma, Y.; Zhang, F.; Zhao, C.; Nie, Y. Insights into the Role of Gut Microbiota in Obesity: Pathogenesis, Mechanisms, and Therapeutic Perspectives. Protein Cell 2018, 9, 397. [CrossRef] [PubMed]

29. Valdes, A.M.; Walter, J.; Segal, E.; Spector, T.D. Role of the Gut Microbiota in Nutrition and Health. BMJ 2018, 361. [CrossRef] 